\title{
Los procesos de juridificación de la ronda campesina de Macusani
}

\author{
Claudia Arredondo Villar \\ Bachiller de Antropología en la Pontificia Universidad Católica del Perú \\ (PUCP). e-mail: claudia.arredondo@pucp.pe
}

\section{Resumen}

El presente artículo explora los procesos de juridificación en las rondas campesinas del distrito de Macusani en la región de Puno, un escenario de pluralismo jurídico presente en los márgenes del Estado. Para ello, se analiza la presencia de elementos del Estado en las prácticas y discursos de los ronderos y ronderas; particularmente, el lenguaje jurídico adoptado por la ronda y lo que ello implica para la construcción de la legitimidad y autoridad de esta organización campesina. Se emplea una metodología cualitativa de corte etnográfico para analizar un caso observado en una asamblea extraordinaria de la ronda de Macusani, en el cual se identifican varios momentos donde se invocan estratégicamente instrumentos legales, que reflejan la adopción y apropiación de discursos y procedimientos para-legales. Entre ellos, se encuentran el Convenio internacional No 169 de la Organización Internacional del Trabajo, la Ley de Rondas Campesinas No 27908, el discurso de Derechos Humanos, y otras normativas del Estado empleadas en los argumentos de los ronderos y su concepción de la justicia.

\section{Palabras clave}

Rondas campesinas, pluralismo jurídico crítico, procesos de juridificación, márgenes del Estado. 


\section{The juridification processes of the peasant round of Macusani}

\section{Claudia Arredondo Villar}

Bachelor in Anthropology at the Pontifical Catholic University of Peru (PUCP). email: claudia.arredondo@pucp.pe

\section{Abstract}

This paper explores the juridification processes that take place in the peasant rounds of the Macusani District in the Puno region, viewed as a place where legal pluralism operates at the margins of the State. For that purpose, elements of the state are identified in the practices and discourse of the peasants. Especially, the legal language adopted by this peasant organization and what that means for the construction of its legitimacy and authority. This qualitative research employs the ethnographic method to analyze a case documented during fieldwork, an emergency meeting of the peasant round of Macusani is narrated, in which several moments where legal instruments are strategically invoked by the peasants are identified. This adoption and appropriation of legal terms and instruments, such as the International Labor Organization's 169 treaty, the $\mathrm{N}^{\mathrm{o}} 27908$ Law of Peasant rounds, the human rights discourse, and other state norms are employed in the arguments of the peasants about what is considered fair.

\section{Keywords}

Peasant rounds, critical legal pluralism, juridification, State margins. 


\section{Introducción}

Muchos ciudadanos necesitan atención de las rondas porque ahora ya saben que somos iguales que juez, que fiscal, que policía. Entonces, más preferencia nos están dando a la ronda, cada vez más vienen más a la ronda que a la policía o ministerio público. Acá ponen denuncias. Por ejemplo, un caso de maltrato familiar, si una señora de Ayapata y un caballero de Macusani tienen problema, ese caso corresponde a la provincial porque son dos distritos diferentes. El presidente de Macusani no puede atender a la señora porque no es su jurisdicción. La ley de rondas bien nos dice que la ronda base de Macusani tiene autonomía en su jurisdicción y, si en su jurisdicción no lo solucionan, nos remiten problema a la provincial. (S. Patatingo, comunicación personal, 11 de mayo del 2018)

En la cita anterior, Segundo, presidente provincial de las rondas de Carabaya, habla sobre la importancia de la ronda en la zona y su reconocimiento como autoridad para administrar justicia; autoridad que actúa de manera equivalente a los agentes del orden del Estado, y es incluso preferida por los ciudadanos sobre la policía. Él pertenece a las rondas campesinas de la zona sur andina, las cuales tienen una importante presencia en el norte de Puno desde la década de los ochenta (Rodríguez, 2007). Esta organización es conocida por su eficacia para combatir el abigeato en el campo, hazaña que sentó las bases para su reconocimiento como la entidad encargada de la seguridad y la administración de justicia en zonas rurales alto andinas. Si bien la ronda nació de las bases, ésta ha probado tener una gran capacidad de organización y movilización que es observable en las redes tejidas a nivel provincial, regional, macro regional (Arenas, 2010), e incluso, nacional ${ }^{1}$.

En las últimas décadas, la ronda se ha consolidado en la sierra sur no solo como un actor importante en la administración de justicia (Rodríguez, 2007; Yrigoyen, 2002), sino también como actor político en la agenda campesina altoandina y en la escena política supracomunal (Arenas, 2010), especialmente a partir de su participación en conflictos sociales (Theidon, 2003) y ambientales (Li, 2015) producidos en los últimos años en el Perú. Además, varios dirigentes ronderos han sido electos para cargos políticos en el Estado; a nivel local, en el puesto de regidor y, a nivel regional, en el cargo de gobernador. Ejemplo de ello es Walter Aduviri, líder aymara, quien ganó las elecciones regionales en octubre del 2018 (Chacón, 2019).

Para entender su sistema de administración de justicia, es relevante considerar el sistema mayor del cual son parte las comunidades campesinas. Las rondas campesinas, por tanto, surgen dentro de este sistema, en el que el Estado es el principal jugador, lo cual tiene un impacto en las prácticas y discursos de la ronda. La cita de Segundo Patatingo nos permite notar esta presencia cuando invoca a la Ley

La Central Única Nacional de Rondas Campesinas (CUNARC) es la organización de rondas campesinas que funciona a nivel nacional. https://www.cunarcperu.com/ 
de Rondas Campesinas y la autoridad que la misma le confiere para tomar decisiones $\mathrm{y}$ hacer justicia en su territorio.

Entonces, surgen las siguientes interrogantes: ¿Qué papel tiene la ley del Estado en las rondas campesinas de Macusani?, ¿cómo interpreta y se apropia la ronda de la ley y otros elementos del Estado?, ¿de qué manera son empleados en favor de la afirmación de la autoridad y jurisdicción de la organización?, ¿cómo es incorporada en los argumentos sobre lo que es justo para los ronderos y ronderas en los espacios de toma de decisión?

\section{Problemática y objetivo de investigación}

Estas interrogantes son particularmente relevantes en un contexto como el peruano en el que, como señala Poole, los estudios antropológicos sobre la ley han estado fuertemente marcados por un enfoque basado en una "dicotomía analítica" que distingue, de un lado, al derecho estatal caracterizado por "la coerción, la inscripción (la escritura) y la parcialidad propias de la soberanía" y, del otro, al derecho indígena, al que se le suele caracterizar como "consensual, oral, y reconciliador." (Poole, 2012, p. 202). Más aún, los estudios sobre la administración de justicia en ámbitos comunales han tendido a privilegiar el análisis de lo que es estrictamente útil para "facilitar y mejorar la administración de justicia" (Poole, 2012, p. 201). Esto, pues, ha invisibilizado a las diferentes voces que, desde estos mismos ámbitos, critican cuestiones fundamentales como los límites al acceso a la ciudadanía, el derecho y la justicia.

Tales aspiraciones aparecieron en las conversaciones con los ronderos y ronderas de Macusani que, si bien valoran el papel de la tradición en sus prácticas de administración de justicia, suelen ligar este interés a una preocupación mayor por el reconocimiento político y el acceso a derechos como ciudadanos. En estos procesos, la ronda usa distintas estrategias para acertar su autoridad y jurisdicción en Macusani; entre ellos, la administración de justicia, para lo cual combina elementos de las costumbres locales con elementos del Estado como, por ejemplo, los instrumentos legales mencionados por Segundo.

En este punto es necesario aclarar lo que se entiende por ley y por justicia. La primera se piensa como un conjunto de normas dictadas por el Estado, por medio de las cuales se regula la vida de las y los ciudadanos. Para ello, un elemento fundamental es la coerción, en tanto la ley implica un castigo o sanción cuando esta no se obedece (Ray, 1996). La segunda, se refiere a la percepción de lograr lo justo por medio de una acción particular, y ello no es necesariamente intrínseco a los elementos de la ley. Ray (1996) incluso argumenta que una vez que lo justo se vuelve ley, la justicia es desplazada, pues que se cumpla una ley no equivale a que se haga justicia. Es por ello importante diferenciar la ley como un conjunto de códigos formales y coercitivos de la justicia, que implica un conjunto de acciones orientadas a lo que 
se considera justo de acuerdo a cada situación y sus particularidades. Siguiendo esta lógica, vale preguntarse cómo es entendida y empleada la ley en las percepciones sobre lo que se entiende como justo para la ronda.

Por lo tanto, este trabajo tiene como objetivo analizar la articulación de los procesos legales de alcance transnacional y nacional con las prácticas y discursos de administración de justicia de la ronda. Además, posee la finalidad de examinar la manera en la que se incorporan elementos del Estado, como la ley, para hacer justicia y reclamar autoridad sobre su jurisdicción.

\section{Metodología}

La presente investigación tiene un enfoque metodológico cualitativo de corte etnográfico (Bolio \& Bolio, 2013; Narváez, 2018) que prioriza el recojo de información a partir de fuentes primarias. Las técnicas implementadas para la investigación fueron entrevistas semi estructuradas a profundidad, conversaciones informales, observación participante y seguimiento de casos de la ronda. El trabajo de campo se realizó en el distrito de Macusani, en la provincia de Carabaya, en la región de Puno en el 2018 como parte del Proyecto "Conflicto, Derecho y Justicia en Comunidades Campesinas, Comunidades Nativas y Rondas Campesinas del Perú" de la ONAJUP. Este tuvo una duración de 3 meses, en los que se acompañó tanto a los dirigentes de la organización de rondas de Macusani como al público que participa en este espacio.

Durante este periodo, se observaron encuentros a nivel distrital (1) y provincial (1), así como asambleas (5) y reuniones (15) de la ronda distrital. Además, se realizaron entrevistas con dirigentes (7), miembros de la ronda (15) y autoridades estatales locales como jueces (2), fiscales (1), defensores del pueblo (2) policías (1), el director de la UGEL (1) y la alcaldía (1). Además, se hizo el seguimiento de tres casos tratados en la ronda. De forma complementaria, se pudo acceder a fuentes secundarias como los archivos del Poder Judicial de Puno y la ronda de Macusani.

El marco interpretativo se sitúa en el debate sobre pluralismo jurídico y rondas campesinas, y para el análisis se toman los conceptos de márgenes del Estado y juridificación. Para ello, se realizó la revisión bibliográfica de los autores que escriben sobre los temas desde diversas disciplinas, tanto en la etapa previa al diseño de la investigación, como posteriormente para el análisis de la data obtenida en campo.

\section{Marco teórico}

El distrito de Macusani se encuentra en el sur andino, donde los procesos de modernización se han traducido en desigualdades económicas, discriminación étnica y exclusión socioeconómica de la población campesina (Burneo y Trelles, 2019), procesos que, en el ámbito de la justicia, se reflejan en la "heterogeneidad jurídica" 
(Feldman, 2010); la cual está marcada tanto por las instancias de justicia comunal, como por la ausencia o débil presencia del sistema judicial del Estado peruano en estas zonas (Gitlitz, 2013; Rodríguez, 2007). En este contexto, tomo la definición de Rodríguez de las rondas campesinas de Puno:

Órganos de vigilancia, protección y de justicia de las comunidades campesinas, ayllus, centros poblados y parcialidades, para ejercer funciones relacionadas a la seguridad comunal, justicia comunal restaurativa, promover el desarrollo comunal, y establecer relaciones de coordinación y diálogo con las instancias del Estado, dentro del ámbito territorial donde se organizan, conforme a los valores, principios y creencias de su propia lógica y racionalidad. $(2007$, p. 16)

Analizo las prácticas de la ronda siguiendo la recomendación de Poole sobre reevaluar "la presencia hegemónica de la ley del Estado dentro de las sociedades y culturas campesinas y nativas" en el Perú (2012, p. 202). En esta línea, la autora propone incorporar una estrategia analítica que permita abordar la ley como un concepto filosófico ambivalente en cualquier ámbito. Tal aproximación implica asumir que la ley no solo establece un contrato social que aspira a ser igualitario, armonioso e inclusivo, sino que, en simultáneo, siempre funda una soberanía "excluyente, violenta y coercitiva" (Poole, 2012, p. 202).

Siguiendo este enfoque, el marco analítico busca distanciarse de los estudios que exponen al Estado como el único administrador racional de la organización política, cuyo alcance y efectividad tienden a debilitarse a medida que se acerca a los márgenes. Y, por el contrario, pretende reflexionar sobre cómo el Estado y la ley son constituidos, ya sea en sus prácticas políticas, regulatorias y/o disciplinarias, desde la vida política que ocurre en sus márgenes (Das y Poole, 2009). El concepto de márgenes del Estado es concebido por Das y Poole mas allá de lo territorial, en tanto "también son, y lo que es quizás más importante, escenarios de práctica sobre los que la ley y otras prácticas del Estado son colonizadas por otras formas de regulación que emanan de las apremiantes necesidades de las poblaciones para asegurar la supervivencia económica y política" $(2009$, p. 8). En esta línea, Davies (2018) considera que la ley puede emerger de esferas distintas del Estado-nación. Entonces, la ley puede ser "abstracta y material, dinámica y estática, determinante y altamente móvil” (p. 23).

El concepto de márgenes del Estado (Das y Poole, 2009) se complementa con los aportes del pluralismo jurídico crítico (Davies, 2018) para intentar re-imaginar a la ley como una práctica enraizada en la vida social, antes que como un conjunto coherente y coordinado de normas o principios que restringen la acción de las personas. Desde este punto de vista, una comprensión pluralista de la ley toma en cuenta la diversidad de los sujetos en sus múltiples y, muchas veces sobrepuestos, papeles como creadores y transmisores de la ley. Solo de este modo es posible visibilizar cómo es que la ley también puede emerger de esferas ajenas a las instituciones del Estado, de sus 
márgenes, y, a la vez, cómo es que la ley estatal, al ingresar en la vida cotidiana de los sujetos que busca regular, no solo los re-configura, sino que al mismo tiempo esta es reconfigurada en este encuentro (Davies, 2018).

En este enfoque encaja el concepto de juridificación propuesto por Sieder, que se refiere a "la invocación estratégica de instrumentos legales y la adopción y apropiación de discursos y procedimientos para-legales por diversos actores sociales" (2010, p. 162) que surgen como "respuesta a la debilidad institucional y las particulares formas perversas de modernización que prevalecen en América Latina, caracterizadas por agudas inequidades socioeconómicas, racismo, y exclusión social y económica de grandes sectores de la población" (2010, p. 162). Esta invocación estratégica de instrumentos legales y cuasi- legales, así como la adopción de discursos y procesos para-legales responde a los déficits de democracia y exclusiones históricas (Sieder. 2010). El concepto para-legal alude a "aquello que se encuentra al margen de", en este caso, la ley. La autora argumenta a partir de los movimientos sociales indígenas en Guatemala que los actores "presentan sus demandas de autonomía no solo o principalmente recurriendo a los tribunales sino por medio de la imitación del Estado y la constitución de (para) legalidades alternativas" (Sieder, 2010, p. 162).

En suma, este estudio se sitúa en el escenario de heterogeneidad jurídica de Macusani, en el que las rondas campesinas, como grupo históricamente marginado, reclama y legitima su autonomía en el ejercicio de la justicia y la soberanía sobre su jurisdicción. Esto se logra por medio de la invocación de principios legales nacionales e internacionales como expone Sieder (2010), y hasta cierto punto, imitando las leyes y procesos del Estado, como el seguir el debido proceso y alinearse al discurso de los Derechos Humanos, las referencias a acuerdos internacionales como el convenio $\mathrm{N}^{\mathrm{o}} 169^{2}$ de la OIT y normativas nacionales como la Ley de Rondas Campesinas $\mathrm{N}^{\mathrm{o}}$ 27908, así como, la importancia que se le atribuye a los documentos escritos; constituyendo así legalidades (para)legales en una relación dialéctica con el sistema jurídico del Estado.

\section{Análisis de caso: Aldo Quispe Quispe ${ }^{3}$}

A continuación, relato un caso presenciado en una de las asambleas de la ronda, al cual hice seguimiento desde la puesta de la denuncia en la oficina distrital de Macusani hasta las discusiones en las reuniones de la ronda. En este caso, identifico elementos que pueden ser interpretados desde los conceptos expuestos en el marco teórico.

\footnotetext{
2 El convenio No 169 de la Organización Internacional del Trabajo sobre Pueblos Indígenas y Tribales reconoce el derecho a la autonomía y el autogobierno en cuestiones relacionadas con asuntos internos y locales. Reconoce el derecho de los pueblos indígenas a conservar y reforzar sus propias instituciones políticas, jurídicas, económicas, sociales y culturales. (OIT, 2014).

3 Este caso fue observado durante el trabajo de campo realizado por la investigadora. Se describe el debate de la asamblea extraordinaria del 3 de abril del 2018 realizada en el local de la Central Distrital de Rondas Campesinas de Macusani.
} 
En el local de la ronda distrital de Macusani alrededor de las 9 de la mañana un miércoles, día que no es usual para las reuniones, comienzan a llegar varios ronderos y ronderas, convocados por el presidente distrital: Antonio. Un hombre llamado Aldo con denuncias por alimentos y reconocimientos de hijos, buscado por la ronda por varios meses ha sido finalmente capturado gracias al dato de uno de los vecinos que lo vio tomando en una cantina. Se encuentra resguardado en un cuarto del local de la ronda mientras se notifica a las partes denunciantes de su captura y se les convoca para una reunión de emergencia. Los miembros de la junta directiva presentes salen en sus motos para convocar a la mayor cantidad de miembros posible y así tener cuórum para el juicio.

En el transcurso de la mañana van llegando más personas, entre ellas, las mujeres denunciantes. El acusado está sentado en una de las bancas del patio junto a una mujer con dos niños y dos hombres del distrito vecino Corani. La mujer es la hermana de una de las ex parejas de Aldo, oriunda de la comunidad campesina de Chacaconiza en el distrito de Corani, quien falleció el año anterior. Acude en representación de su sobrino, un pequeño de 2 años, para quien demanda se pague una suma de dinero por alimentos. Un tiempo después, llegan dos mujeres con sus hijos en la espalda, una de ellas le reclama en quechua, le entrega a su hijo bruscamente y le pega entre gritos e insultos. Aldo comienza a responderle entre gritos clamando: "no es mi hijo, más bien cuida a mi hija, cochina la mandas al colegio" hasta que Corina, secretaria de la junta directiva, les llama la atención "estamos en el local de la ronda, ¡no se puede meter mano!, vamos a hablar en asamblea". Las otras mujeres comienzan a gritarle de nuevo al acusado, por lo que los ronderos deciden regresarlo al cuarto, aislado de los demás, hasta que comience la asamblea extraordinaria.

Van llegando más ronderos y ronderas y se van formando grupos distribuidos por el patio del local. Algunos están apurados por comenzar, temen que el proceso demore dado que los casos son de distintas bases y quieren solucionarlo el mismo día. El presidente tiene que hacer la solicitud para empezar y tiene que escribirla el secretario, Elmer, pero no está presente. Los asistentes hacen hincapié en el debido proceso en respuesta a los que protestan por comenzar, aclaran que se debe tener una cantidad considerable de ronderos y ronderas en la asamblea y todas las partes deben tener el tiempo necesario para presentar su caso. Finalmente, alcanzan un número adecuado, alrededor de 50 personas, y entran todos al salón de asambleas.

El presidente de la ronda distrital se sienta en la mesa del frente rodeado de los otros miembros de la junta directiva, el secretario de actas abre el cuaderno de actas y comienza a anotar los nombres de los y las presentes. El resto de asistentes se acomodan en las sillas ubicadas al frente y a los lados de la mesa de la junta directiva. Las mujeres denunciantes presentes se sientan junto a los presidentes de sus respectivas bases, quienes las acompañan y representan en la asamblea. Dos ronderos traen a Aldo y lo ponen en el centro del salón, entre la junta directiva y el público. 
Primero, presentan el caso de María, hermana de la difunta Yoana, que tuvo un hijo con Aldo en la comunidad campesina de Chacaconiza, Corani. Tanto los ronderos de la base de la comunidad campesina como de la base del distrito la acompañan, pues ambos han visto su caso y en coordinación con la base del distrito de Macusani han estado buscando al acusado. Aldo ya se había comprometido anteriormente a pagar la manutención de su hijo, pero nunca cumplió. Los representantes de la base de Chacaconiza leen el acta frente a todos los presentes especificando los términos del trato y luego la pasan para que los que deseen la examinen con más detalle. Comienza el debate. El presidente de la base de Miraflores, Eliseo, opina "este hombre no tiene nombre para mí. ¿cómo es posible que tenga tantos hijos si no los va a mantener? Si no te vas a encargar ¿para qué tienes tantos? Tiene que pagar pensión alimentaria, tiene que comprometerse a pagar mensual". Lidia, presidenta de la base de Hatun Phinaya, agrega "Vergüenza deberías sentir. Que pague los estudios de su hija hasta los $18 \mathrm{y}$ si quiere ir a la universidad hasta los 25. ¡Encima no es la única mujer que lo denuncia!". Aldo se encuentra en el centro de las miradas, visiblemente nervioso y cabizbajo ante los comentarios.

Pide la participación Manuel, uno de los dirigentes más antiguos, y con un peluche en la mano resondra a Aldo, dice que el castigo debe dolerle, tiene que encargarse de sus hijos, no se puede escapar de esa responsabilidad. Agrega que "la ronda puede ejercer el derecho consuetudinario, según la OIT 169 y la ley de rondas nosotros podemos sancionar. Es un delito abandonar a los hijos. No es posible que escape de sus responsabilidades, se esconde de las madres de sus hijos. ¡A la disciplina!". Le toca a Aldo explicarse, pero se ve muy alterado y solo atina a decir que no tiene trabajo estable, ni familia a la cual recurrir, que vive en el campo con su madre que es mayor, y si consigue trabajo le puede dar 100 soles a su hija. De nuevo protesta Manuel "100 soles no alcanza para nada, ahora vas a tener que trabajar día y noche para mantener a tus hijos. El niño come, estudia, no sabes cuánto cuesta la mensualidad, los libros, el micro. Las wawas tienen derechos, tienen que estudiar, necesitan su alimentación y salud. Así como hay derechos humanos, hay derechos del niño. Tiene que haber acta de compromiso, que pague 100 soles mensual, y después que pague más". El presidente de la ronda, escogido como presidente del debate, pregunta si todos están de acuerdo con el pago mensual de 100 soles.

María acepta y los ronderos de Corani y Chacaconiza registran todo en sus respectivos cuadernos de actas. Mientras lo rotan por todos los y las presentes para que registren sus sellos y firmas en signo de conformidad con la decisión registrada en los documentos, algunos le dan consejos a Aldo. Corina le dice que los hombres pueden conseguir trabajo porque hay más para ellos, que se vaya a buscar fuera de Macusani, a las minas. Antonio, el presidente, le dice que no puede ir por todos lados teniendo hijos, tiene que asumir, además puede contagiar enfermedades. Le pide que reflexione y recapacite, que vaya a ver a sus hijos, les de manutención y se ocupe de su educación. Muchos no están de acuerdo con la suma, siguen diciendo que es muy poco, por lo que se decide, confiscar la moto de Aldo en parte de pago, aparte 
de los 100 soles mensuales a los que se ha comprometido. Con esto los ronderos de las bases de Corani y Chacaconiza le agradecen a la base de Macusani y se retiran.

El segundo caso se trata de Rojelia, ex pareja de Aldo, con quien tiene 3 hijos. Ella se presenta acompañada del presidente de la base del barrio Jorge Chávez, Macusani, quienes proceden a leer las actas de denuncia y asambleas anteriores realizadas a nivel de su base. En ellas, Rojelia exige que Aldo se haga cargo de sus hijos, y que, además, reconozca la paternidad del último de ellos, un menor de 3 años.

Le piden a Rojelia su versión de los hechos, ella cuenta que se separó de Aldo hace unos años porque era un hombre violento, que tomaba mucho y a veces le pegaba. En una ocasión le dijo que la iba a matar si no quería tener sexo con él, que la amenazó y su último hijo es producto de esa violación. Por eso reclama que lo reconozca y pague la manutención por todos sus hijos. Aldo responde y se defiende argumentando que nunca la amenazó. Se separaron hace 4 años en el barrio Túpac Amaru, hay un acta de separación de cuerpos y de separación de bienes. Admite que en una fecha llegó borracho y hubo violencia, pero dice que ella también se ha portado mal, que no cuida bien a sus hijos, que una vez mandó a su hija con la camisa sucia al colegio. Se niega a reconocer al menor.

El presidente manda a uno de los ronderos a la base del barrio Túpac Amaru para que pida el acta de separación de la que habla Aldo. Mientras esperan, participa Nélida y se dirige primero a Rojelia: "debiste hacerlo firmar hace tiempo. Tu hijo tiene ya 3 años ¿por qué reclamas ahora?" Segundino, el vicepresidente de Macusani, interviene a continuación y habla sobre el valor de la familia y el deber de hacerse cargo de los hijos. Lo reprime por su irresponsabilidad y falta de valores cristianos. En paralelo, Rojelia y Aldo que han estado intercambiando palabras por lo bajo, comienzan a gritar y ella intenta pegarle. Manuel se para en medio de los dos para evitar la pelea y llama al orden, agrega que es mejor esperar al acta de la base del barrio de Túpac Amaru, pues cada base tiene autonomía y no pueden tomar una decisión sin antes leerla y saber que se había decidido anteriormente. Hace énfasis en que no se puede juzgar dos veces, pues hay que seguir el debido proceso.

Después de unos cuarenta minutos de debate llega el rondero respirando agitadamente con el acta en la mano. Manuel procede a leerla en voz alta al medio del salón, parado entre Rojelia y Aldo, “...Se acusa a Aldo Quispe Quispe de agresión física y psicológica...Por decisión voluntaria de ambas partes se hará separación de cuerpos...Los hijos quedan con la madre y la hija con el padre. El ganado de 30 alpacas, 25 llamas y 20 ovinos quedan para la mujer. Su padre cuidará del ganado. De no cumplir con esta separación la sanción es de 5 mil adobes entre ambas partes....". Ahora el debate vira hacia el cumplimiento del acta y la sanción. Una rondera de la base de Pacaje, Juana, interviene "no han cumplido con el acta y deben cumplir con la sanción. Se tiene que respetar la decisión de la ronda. Si no existiera esa acta otra sería la historia, pero ya hay acuerdo previo y se tiene que cumplir con eso", luego 
Eliseo pregunta "¿para qué reclama? Si ese hijo fue concebido después de acordar en la ronda y firmar el acta no tiene derecho a reclamar. Si no va a cumplir con el acuerdo, ¿para qué viene a la ronda? Nos está haciendo perder el tiempo, no hay respeto a la ronda". Corina responde en defensa de Rojelia que Aldo la obligó a tener relaciones con él haciendo uso de la fuerza y que "debe ser castigado por violencia doméstica, porque agresión física y psicológica es violencia doméstica y eso es delito. Eso ha enseñado el fiscal en el encuentro provincial, eso hay que denunciar. La violencia doméstica es problema grande. No podemos dejar pasar esas cosas".

Manuel interviene de nuevo y demanda que se cumpla la sanción. Él es tío de Rojelia por matrimonio, pues es la sobrina de su esposa, pero asegura que no habrá favoritismo y se compromete en su nombre a que cumpla con la entrega de los adobes que le corresponden: 2500. Luego los llama a la disciplina. Aldo y Rojelia ya en el centro del salón, se arrodillan uno al lado del otro y bajan las cabezas. Los ronderos y ronderas proceden a participar, algunos los reprimen, otros los aconsejan, unos hablan de Dios y los valores cristianos de la familia, otros hablan de la ley y los delitos de violencia doméstica y abandono de los hijos.

Después de una larga discusión se decide que Aldo debe reconocer al niño y que ambos deben cumplir con la sanción de 5 mil adobes por no cumplir con el acuerdo del acta de la base Túpac Amaru, 2500 cada uno para el 31 de julio. El 1 de agosto el presidente debe informar que se ha cumplido con la sanción, de lo contrario los llevarán a la cadena ronderil. Manuel de nuevo se compromete en nombre de Rojelia y dice que por ser su ahijada le ayudará pagando por los materiales y supervisará que ella los haga, pero por Aldo no se puede responsabilizar. Antonio sigue aconsejando a Aldo, le dice que se responsabilice por sus acciones, que no se pelee con su mujer y que mantenga a sus hijos. Luego Lidia objeta a que Aldo se quede con la hija, de acuerdo al acta de la base Túpac Amaru esa fue la división, pero le responden que eso ya es cosa juzgada y lo decidido por esa base y registrado en el acta debe cumplirse. Es el debido proceso.

El secretario de actas procede a redactar la decisión mientras que Manuel administra la disciplina a Rojelia. Él puede hacerlo porque es su familiar, primero se acerca a Rojelia y le habla suavemente, aconsejándola para no volver a caer en el mismo error, a continuación, mientras ella sigue de rodillas Manuel invoca en su discurso al creador, a los apus, a la organización de las rondas y a sus antepasados antes de darle 3 chicotazos en las piernas, en un acto simbólico de corrección, como el de un padre a un hijo. Sigue Aldo, a quien nadie le pega porque no tiene ningún familiar presente y nadie se ofrece a darle con el chicote porque temen ser procesados por agresión. Muchos ronderos y ronderas han tenido problemas con la justicia ordinaria por aplicar castigos físicos. Por eso, resaltan que debe ser un familiar, sino puede ser visto como violación de derechos humanos. Manuel, aun con el chicote en la mano, agrega que no quiere ser procesado de nuevo, pues ya tiene una denuncia por un desalojo de terreno que fue decisión de la ronda distrital y ya no quiere más problemas con la ley. 
Entonces, a Aldo lo aconsejan también, le dice que debe cumplir con sus compromisos, que debe darles un buen ejemplo a sus hijos y le piden unirse a la ronda, "caminar con ellos", y aprender a ser un hombre responsable y disciplinado, además ellos lo pueden ayudar a conseguir trabajo. Algunos siguen comentando que si no cumple el acta lo mandan a cadena ronderil y varios opinan que Aldo es muy problemático y necesita un castigo mayor. Las conversaciones siguen en el salón de asambleas mientras se registran los acuerdos. Finalmente, Elmer termina de escribir el acta de la asamblea y rota el cuaderno por el salón para que todos los presentes la firmen y sellen en signo de conformidad, legitimando así los acuerdos pactados y dando fin a la reunión.

¿Cuál es la imagen de la ley que emerge a partir de esta historia y qué implica para la organización de las rondas campesinas de Macusani? ¿Qué nos dice sobre la relación entre el derecho internacional, el marco legal institucional del Estado y el sistema de justicia de la ronda? ¿Qué podemos inferir sobre la autoridad y la jurisdicción de la ronda? Nos encontramos en un escenario de heterogeneidad jurídica (Davies, 2018) en el que podemos ver cómo se encuentran las leyes del Estado enraizadas en el discurso de los ronderos. En este caso se puede ver cómo el discurso legal que provee los lineamientos para los delitos de violencia doméstica y sexual, así como los derechos de los niños, se manifiesta en los espacios de administración de justicia de la ronda. Dichos lineamientos son transmitidos por las autoridades estatales; en este ejemplo, por el fiscal regional en un encuentro provincial, y asimilados, interpretados y apropiados por los ronderos dentro del sistema de justicia comunitario. De igual manera, el discurso de los Derechos Humanos ha sido transmitido por actores del Estado como jueces y fiscales, así como, por organizaciones sin fines de lucro y la iglesia en el último par de décadas. La asimilación y apropiación de estos lineamientos legales nacionales e internacionales se evidencian en varios de los argumentos de los ronderos y ronderas durante la discusión sobre el caso de Aldo.

Estas cualidades no son exclusivas de la ronda de Macusani. Piccoli (2011) encuentra situaciones similares en Cajamarca. La autora nota que, si bien el pluralismo jurídico contiene contradicciones y tensiones difíciles de resolver teóricamente, los actores locales las adaptan de manera pragmática. Dichas adaptaciones se observan en las negociaciones que se dan en la práctica cuyo diálogo tiene lugar en los márgenes de los dos sistemas jurídicos, y refleja así la pluralidad de sistemas de justicia. En ellos, los actores (jueces, policías, ministerio público y ronderos) elaboran estrategias que pueden parecer transitorias, al asegurar de manera relativamente durable la coexistencia de la justicia campesina en el seno del sistema legal peruano (Piccoli, 2011). Dentro de estas estrategias, se encuentra la incorporación de las leyes del Estado en los discursos de la ronda, tanto para la justificación de sus decisiones como para la legitimación de sus acciones.

Podemos notar, en primer lugar, en el argumento de Corina que aprendió que la violencia física y psicológica son violencia doméstica y deben denunciarse en uno de los encuentros de la ronda donde invitaron al fiscal regional. De igual manera, Manuel habla sobre los derechos de los niños y los Derechos Humanos en su argumentación 
para el reconocimiento de los hijos y el pago de alimentos. Ambos están invocando discursos legales en los espacios de toma de decisión de la ronda con el objetivo de justificar sus percepciones sobre lo que es justo. En este caso, que Aldo se haga cargo de sus hijos y sea castigado por violencia física, psicológica y sexual.

En segundo lugar, el discurso de Derechos Humanos tiene importantes repercusiones en las prácticas de la ronda. Debido a los procesos judiciales por los que han pasado varios líderes ronderos, como usurpación de funciones, secuestro, violación de los derechos fundamentales, entre otros (Picolli, 2011; Rodríguez, 2007), las prácticas de sanción y corrección de la ronda han cambiado. En sus inicios, la ronda campesina de Macusani imponía castigos drásticos como arrodillarse sobre espinas, meterse al río en bajas temperaturas y varios golpes con chicote. Sin embargo, en los últimos años, en respuesta a los problemas con la justicia ordinaria, los castigos constan principalmente de ejercicios físicos y multas.

El violador nos ha denunciado a todos los ronderos. Mi persona ni siquiera estaba presente. yo no sabía cómo le han detenido todo eso, y hemos recibido una denuncia por tortura y secuestro. Hicimos archivar el caso porque nunca le había metido la mano, porque yo respeto mucho los derechos humanos y no soy capaz de tocar a las personas. (E. Cuno, comunicación personal, 19 de abril del 2018)

La abstención de castigar de manera física a Aldo es justificada de la misma manera, con la intención de evitar ser procesados por la justicia ordinaria. Mientras que Manuel le da los tres chicotazos a Rojelia, nadie lo hace con Aldo, porque no hay ningún familiar presente. Esta relación es importante para realizar este acto, porque, de acuerdo a las explicaciones de los ronderos, eso los protege de ser acusados y procesados por la justicia ordinaria por delitos como agresión.

En tercer lugar, otras leyes que los eximen de ser procesados y a través de las cuales se justifican y se reconocen sus acciones como legítimas son el convenio $\mathrm{N}^{\circ} 169$ de la OIT y la Ley No 27908 de Rondas Campesinas. Como vemos, en el discurso de Manuel estas les reconocen el derecho a la autonomía y el autogobierno, lo cual incluye la esfera jurídica.

He visto casos de ronderos dirigentes por secuestro, tortura, privación de libertad, pero esos casos generalmente se archivan porque los ronderos van al poder judicial y la fiscalía y presionan, usan como argumento el artículo 149, la OIT 169, el acuerdo plenario del 2011, y se archiva. (V. Vilca, comunicación personal, 11 de abril del 2018)

Los dirigentes de la ronda constantemente resaltan las facultades que la Ley de Rondas Campesinas $\mathrm{N}^{\circ} 27908$ aprobada en el 2003 les otorga al reconocer su jurisdicción, y algunos dirigentes indican que pueden ejercer las mismas funciones que un fiscal. Por ejemplo, ordenar un levantamiento de cadáver: 
La ronda puede investigar, ayudar, ¿qué pasó?, ¿quién vio? El presidente de comunidad puede hacer levantamiento de cadáver, tiene autonomía. El presidente de la comunidad o la ronda llama al fiscal para hacer la necropsia coordinando. El fiscal está obligado a escuchar a la comunidad”. (F. Lope, comunicación personal, 3 de mayo de 2018)

En el caso de Aldo vemos cómo Manuel defiende la autoridad de la ronda para decidir sobre el castigo de Aldo y la compensación a las mujeres denunciantes, invocando el reconocimiento legal de las rondas campesinas en la ley $\mathrm{N}^{\mathrm{o}} 27908$ y al tratado internacional $\mathrm{N}^{\circ} 169$ de la OIT. Es por medio de estos recursos legales que la ronda reclama autonomía en la toma de decisiones dentro de su jurisdicción.

En cuarto lugar, se encuentra el debido proceso para la administración de justicia. Éste involucra un conjunto de normas, pasos y elementos que deben ser cumplidos para que las decisiones y acciones de la ronda sean consideradas legítimas. En varios momentos del caso expuesto se menciona el debido proceso: en la lectura de las actas, en la cantidad del público necesario para comenzar la asamblea, en la importancia de la denuncia, la forma de presentación de los casos por medio de documentos. En ambos casos relatados se puede observar que la justicia de la ronda sigue un proceso de diversas etapas.

\section{Figura 1}

El debido proceso.

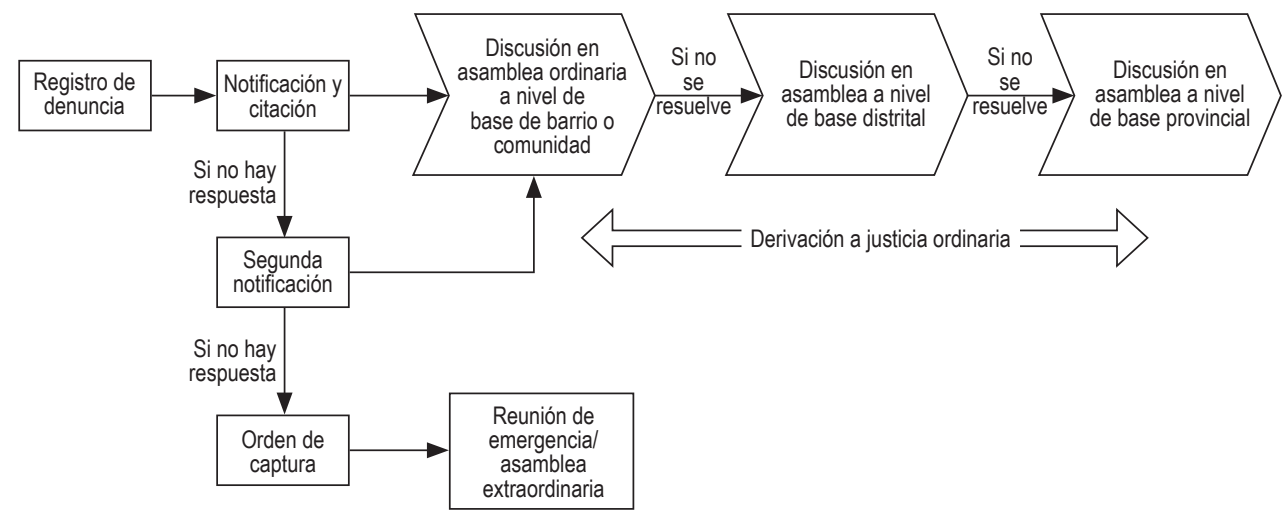

Nota: Elaboración propia

En la imagen podemos ver a grandes rasgos cómo funciona el proceso de la justicia que debe seguir la ronda. El primer paso siempre es la denuncia en la base de la comunidad campesina o del barrio, la cual debe ser reportada en la oficina de la ronda a algún miembro de la junta directiva y registrada en el cuaderno de denuncias.

Primero siempre en la base, la base es una partecita nomas, digamos Túpac Amaru. Si es en ahí la denuncia, de ahí empieza. En ahí deberían solucionar, si no soluciona, ya pasa a distrital. Pero de ahí siempre es la que nace, de una 
denuncia. Pero no puede venir directo a distrital. Siempre va a ser de alguna parte, y, además, no puede ir de acá por ejemplo de Túpac Amaru a recibir esa denuncia del otro lado, porque eso ya tiene su sitio. (J. Quispe, comunicación personal, 16 de marzo del 2018)

A continuación, se notifica a la persona acusada para citarla a una asamblea en la que se discutirá la denuncia. En caso no haya respuesta, se manda una segunda notificación y si la parte acusada sigue sin responder, se le pone una orden de captura y se convoca a una reunión de emergencia. Tal es el caso de Aldo, quien al no responder a las notificaciones de la ronda, fue capturado y juzgado en una reunión de emergencia o asamblea extraordinaria. Idealmente, los casos deben solucionarse a nivel de la base de la comunidad o del barrio, pero cuando no se puede llegar a una decisión se deriva el caso al siguiente nivel: primero al distrital y finalmente al provincial. La ronda a nivel regional ve asuntos relacionados a políticas de la región, rara vez trata casos específicos. En cualquiera de estas etapas y si la mayoría así lo decide, el caso puede ser derivado a la justicia ordinaria.

Es interesante comparar esta figura del debido proceso con la practicada por el sistema jurídico del Estado, en el que "el debido proceso encierra en sí un conjunto de garantías constitucionales que se pueden perfilar a través de identificar las cuatro etapas esenciales de un proceso: acusación, defensa, prueba y sentencia, que se traducen en otros tantos derechos" (Landa, 2002, p. 448). Tanto el sistema de administración de justicia de la ronda como el del Estado están pautados por un "debido proceso", que es necesario para que se reconozca la administración de la justicia como legítima.

Uno de los elementos que pertenece al debido proceso y aparece en el caso narrado son las actas, en ellas se plasman las decisiones que tienen lugar en las asambleas como parte fundamental del proceso de la justicia comunitaria. Los ronderos y ronderas hacen hincapié en la importancia de tener las actas legalizadas por el notario y contar con las firmas y sellos de todos los asistentes en las asambleas. De esta forma, las decisiones tomadas respecto a los acuerdos son validadas y, en caso los dirigentes de la ronda sean denunciados en el sistema de justicia estatal, las actas sirven como prueba de la legitimidad de las resoluciones dictaminadas por la ronda. Nuevamente se observa cómo están presentes las prácticas del Estado.

El acta de arreglo legaliza. Debe decir "de la denuncia se ha arreglado tal fecha, por ejemplo, si vuelve a engañar se compromete a hacer 500 adobes". El padrón se abre cada dos años, tienen que legalizar en el notario con los sellos y las firmas, con la impresión del notario y estar en los registros. El libro del padrón y las actas se debe actualizar, llevan a legalizar al notario cada dos años. (J. Quispe, comunicación personal, 16 de marzo de 2018)

El castigo de Aldo y Rojelia era hacer 5000 adobes por romper el acuerdo registrado en el acta de separación de la base del barrio Túpac Amaru. Este documento encarna 
la decisión colectiva tomada por la ronda y posee la autoridad para hacer que se cumpla con el castigo acordado. La existencia del acta de separación influye en el argumento de negar el derecho de Rojelia al reconocimiento de su hijo debido al acuerdo previo de separación.

La importancia de estos registros, encarnados en las actas de la ronda, puede ser leída desde la visión de artefactos gráficos de Hull (2003). De acuerdo al autor los documentos no son solo instrumentos de las organizaciones burocráticas, sino que son estos, en sí mismos, constitutivos de reglas burocráticas, ideologías, conocimientos, prácticas, subjetividades, objetos y resultados. Es a través de ellos que se canaliza la agencia de múltiples individuos y se manifiesta la agencia colectiva (Hull, 2003). Bajo esta lógica, los artefactos gráficos no son simples instrumentos de una organización social, sino que ellos mismos, por medio de la canalización de la agencia colectiva, participan en la formación de redes y grupos tanto dentro como fuera del sistema burocrático (Hull, 2003).

En este caso, el acta de acuerdo de separación, y el poder que tiene en sí misma para imponer las decisiones de la ronda, es un artefacto gráfico que existe fuera de la burocracia del Estado, pero que, a su vez, lo imita para legitimar su autoridad. Además, el carácter colectivo que representa el documento protege a los ronderos y ronderas de ser juzgados por la justicia ordinaria al eximirlos de la responsabilidad individual. Este uso de artefactos gráficos (Hull, 2003) podría ser comparado a una de las particularidades del derecho del Estado: la inscripción, que dentro de la dicotomía señalada por Poole (2012) pertenecería a la soberanía del mismo. Su incorporación en los discursos y prácticas de la ronda cumple el objetivo de legitimar los acuerdos y sanciones.

Los elementos expuestos ayudan a comprender los procesos de juridificación en el sentido descrito por Sieder (2010), es decir, aquellas circunstancias en las que diferentes actores invocan estratégicamente una serie de instrumentos legales y/o adoptan y se apropian de discursos y prácticas que simulan procedimientos legales (Sieder, 2010). En ese sentido, la ronda campesina de Macusani, como actor externo al Estado, incorpora en su organización discursos y prácticas del Estado e imita procedimientos legales.

En primer lugar, emplean el lenguaje jurídico del Estado para argumentar que la violencia física, psicológica y sexual son delitos, y que el pago de alimentos es una obligación del padre. En segundo lugar, el discurso internacional sobre los Derechos Humanos es familiar en las discusiones de la ronda, pues se plantea como la justificación legal de los procesos judiciales de los que han sido partícipes varios dirigentes y miembros de la organización, y a su vez, ha sido inculcado por organizaciones internacionales, representantes del Estado y la Iglesia en las rondas campesinas a lo largo de las últimas décadas con el objetivo de evitar ciertas prácticas que se consideran como violación de estos derechos. En tercer 
lugar, la invocación a tratados internacionales como el convenio $\mathrm{N}^{\mathrm{o}} 169$ de la OIT y normativas legales nacionales como la Ley No 27908 de Rondas Campesinas para el reclamo de autoridad y autonomía en la toma de decisiones de la ronda. Y, finalmente, el cumplimiento del "debido proceso" para administrar justicia de manera adecuada y legítima.

A partir de los ejemplos mencionados podemos decir que, además de los usos reivindicativos del aparato judicial que, como sabemos, no han sido ajenos a las comunidades y organizaciones indígenas ante su imposibilidad de canalizar sus demandas históricas a través de procesos políticos (Méndez, 2005) ${ }^{4}$, los procesos de juridificación reivindican el derecho de los campesinos de Macusani a la jurisdicción jurídica, económica y política a través de la imitación del funcionamiento del Estado, constituyendo así "(para) legalidades alternativas" (Sieder, 2010). La inclusión del debido proceso y elementos como el libro de actas, firmas y sellos para legitimar las decisiones, así como la presencia del discurso de Derechos Humanos, y la incorporación de delitos como violencia doméstica, física, psicológica y sexual, y de deberes como pago de alimentos en la argumentación de los casos, muestra la asimilación y apropiación de los instrumentos legales del derecho nacional e internacional por parte de la ronda de Macusani.

Como se ha ilustrado, las leyes del Estado y discursos internacionales sobre Derechos Humanos han calado en la forma de hacer justicia de la ronda, siendo estos incluidos, interpretados y apropiados en los espacios y prácticas de la ronda, contribuyendo así a la construcción y legitimación de su autoridad en Macusani. En este sentido, es evidente que la fuerza de la ley no proviene exclusivamente del poder soberano del Estado, sino que los alcances de esta son debatidos, y legitimados en el espacio de la ronda. Los ronderos no son simples "benefactores pasivos" de una ley estatal que les promete justicia a partir de normas y sanciones que protegen sus derechos (Poole, 2012, p. 223), sino que, por el contrario, en Macusani, las normas locales son las que establecen los alcances y criterios de justicia, a la vez que estas son implementadas apelando a la ley del Estado.

\section{Conclusiones}

El dominio de lo consuetudinario en Macusani, y las interpretaciones de la ley del Estado nos dirigen a pensar tanto en las posibilidades que brindan estos espacios para la comprensión de la ley fuera de los espacios institucionales del Estado, como también en las posibilidades que otorga al Estado para la gobernanza del territorio andino. La ronda ha probado ser una organización con vocación para establecer

\footnotetext{
4 Méndez (2005) rastrea la participación política de los campesinos de Ayacucho por medio de registros que datan desde la época colonial, centrándose en la rebelión de 1820. La autora desmitifica la imagen del campesino atrasado y alejado de las luchas políticas del país, usualmente presentada en la literatura, $y$, en cambio, ilustra la imagen de los campesinos como agentes activos que participan en los procesos políticos por otros medios, entre ellos la guerra. La imagen del campesino como agente activo en su propia historia encaja con la figura de autoridad que proyecta la ronda campesina.
} 
la regulación colectiva de la vida en su territorio cuyo alcance cubre diversas dimensiones: desde el ámbito privado familiar hasta la seguridad comunal y distrital. Si bien esta organización se caracteriza por tener constantes roces con el Estado, también actúa como una figura de autoridad local que asegura el cumplimiento de la ley.

Por ejemplo, en el contexto de la pandemia de la COVID-19 las rondas campesinas han probado ser valiosos aliados para el cumplimiento de las medidas de seguridad, al asumir así la función de control territorial. La ronda asegura el cumplimiento del toque de queda y el control de la movilidad por medio de la revisión de documentos y el llamado de atención a las personas que no cumplen con las medidas de emergencia impuesta por el Estado.

La voluntad de los ronderos en respaldar la bandera de la vida y la salud en sus respectivas jurisdicciones ha contribuido a que el estado de emergencia se respete. Hugo Tapara Saya, vicepresidente de la Cunarc-p (Comunicación FILAC, 2020)

La ronda toma la ley del Estado, la apropia y la refuerza en el ámbito local. De esta manera, la ley emerge en una esfera distinta a la estatal, se manifiesta e interpreta en la cotidianidad de los campesinos de Macusani; particularmente, en los espacios de la ronda, considerados por largo tiempo como marginales. Como se puede ver en el caso narrado, diversos instrumentos legales como las leyes con respecto a un delito de violencia física, psicológica, doméstica y la obligación del pago por alimentos, así como, la mención de convenios internacionales como la OIT 169 y nacionales como la Ley de Rondas Campesinas que reconocen su derecho a la autonomía y el autogobierno, aparecen en los argumentos de los ronderos y ronderas. Ellos cumplen, entonces, un rol activo como creadores y transmisores de la ley. Por medio de la interpretación y apropiación de esta, la ronda establece su autoridad para decidir sobre la vida de las personas que habitan en su jurisdicción. Además, se expande a esferas no contempladas por la ley estatal como la infidelidad, problemas de pareja, las disputas entre vecinos y la cobranza de deudas.

En conclusión, los ámbitos de la justicia ordinaria y la justicia de la ronda son contiguos, no solo en términos espaciales, sino también en lo jurídico y político. En el caso analizado vemos como la ronda toma las prácticas jurídicas del Estado y las hace suyas por medio de la mímica con el propósito de evocar su poder, estableciendo así su autoridad y la legitimidad de su jurisdicción política y jurídica en el distrito de Macusani. Las rondas demuestran que no son actores pasivos en este escenario apelando constantemente al reconocimiento de la ley estatal, a normas y estructuras de autoridad local, y a instrumentos e instituciones transnacionales; estableciéndose de esta manera como figura de autoridad y regulación en Macusani y reclamando así incidencia en las decisiones políticas y jurídicas que competen a la población que habita su territorio. 


\section{Referencias bibliográficas}

Arenas, F. (2010). Institucionalización democrática de los conflictos sociales en el sur andino: El caso de las rondas campesinas de la provincia de Carabaya. Lima: CIES, CBC.

Bolio, J \& Bolio, H. (2013). El método cualitativo etnográfico y su aplicación para los estudios jurídicos. Revista Logos, Ciencia \& Tecnología, (2), 158-165.

Burneo, M \& Trelles, A. (2019). Comunidades Campesinas en Puno y nueva ruralidad. Puno en el siglo XXI: Desarrollo, ambiente y comunidades (pp. 183 - 238). Lima: SER.

Chacón, K. (2019, 7 de enero). El primer dirigente aymara que asume como gobernador regional de Puno. Ojo Público. https://ojo-publico.com/1042/el-primer-dirigente-aymaraasume-la-presidencia-regional-de-puno

Congreso de la República del Perú. (2003, 20 de diciembre). Ley de Rondas Campesinas N. 27908. Diario Oficial El Peruano. https://docs.peru.justia.com/federales/leyes/27908jan-6-2003.pdf

Comunicación FILAC. (2020, 25 de mayo). La valiosa labor de las rondas campesinas en la lucha contra el coronavirus. FILAC. https://www.filac.org/wp/comunicacion/actualidadindigena/la-valiosa-labor-de-las-rondas-campesinas-en-la-lucha-contra-el-coronavirus/

Das, V. \& Poole, D. (2009). Anthropology in the margins of the state. Santa Fe: School of American Research Press.

Davies, M. (2018). Law unlimited: Materialism, pluralism and legal theory. Abingdon: Routledge.

Feldman, I. A. (2010). Heterogeneidad jurídica y violencia fundacional. Todas las sangres. Revista De Crítica Literaria Latinoamericana, (72), 233-252.

Flores Galindo, A. (1988). La imagen y el espejo: la historiografía peruana 1910-1986. Márgenes. Encuentro y debate, 2(4), 55-83.

Gitlitz, J. (2013). Administrando justicia al margen del Estado: Las Rondas Campesinas de Cajamarca. Lima: IEP.

Hull, M. (2003). The file: agency, authority, and autography in an Islamabad bureaucracy. Language \& Communication (23), 287 - 314. doi:10.1016/S0271-5309(03)00019-3

Landa, C. (2002). El derecho fundamental al debido proceso y a la tutela jurisdiccional. Pensamiento Constitucional. 8. (8), 445 - 461. Fondo Editorial de la Pontificia Universidad Católica del Perú

Li, F. (2015). Unearthing conflict: corporate mining, activism, and expertise in Peru. Durham: Duke University Press. 
Méndez, C. (2005). The plebeian republic: The Huanta rebellion and the making of the Peruvian state, 1820-1850. London: Duke University Press.

Narváez, R (2018). La etnografía: instrumento de investigación en antropología jurídica. El caso de un pueblo amazónico. Revista de temas sociológicos (23), 307 - 341.

Organización Internacional del Trabajo. (2014). Convenio Núm. 169 de la OIT sobre Pueblos Indígenas y Tribales. Declaración de las Naciones Unidas sobre los Derechos de los Pueblos Indigenas. Lima: OIT/Oficina Regional para América Latina y el Caribe.

Piccoli, E. (2011). Vie paysanne et gouvernance rondera dans les Andes: Ethnologie de la communauté de l'Ahijadero et de la gestion du vivre ensemble a Bambamarca, Pérou. Lovaina: Universidad Católica de Lovaina.

Poole, D. (2004). Between threat and guarantee: justice and community in the margins of the Peruvian state. Anthropology in the Margins of the State.

Poole, D. (2006). Los usos de la costumbre: hacia una antropología jurídica del Estado neoliberal. Alteridades, 16 (31), 9-31.

Poole, D. (2012). La ley y la posibilidad de la diferencia: la antropología jurídica peruana entre la justicia y la ley. No hay país más diverso II. Compendio de antropología peruana (pp. 199-245). Lima: IEP.

Ray, T. (1996). Differentiating the Related Concepts of Ethics, Morality, Law and Justice. New directions for teaching and learning, (66), (47-53).

Rodríguez, C. (2007). Justicia comunitaria y Rondas campesinas en el sur andino. Lima: SER.

Sieder, R. (2010). Legal Cultures in the (Un)rule of Law: Indigenous Rights and Juridification in Guatemala. Cultured of Legality: Judicialization and Political Activism in Latin America (pp. 161-181). Cambridge: Cambridge University Press.

Theidon, K. (2003). Disarming the Subject: Remembering War and Imagining Citizenship in Peru. Cultural Critique, (54), 67-87.

Yrigoyen, R. (2002). Hacia un reconocimiento pleno de las rondas campesinas y pluralismo legal. Allpanchis, 34. (59/60), 31-81. 